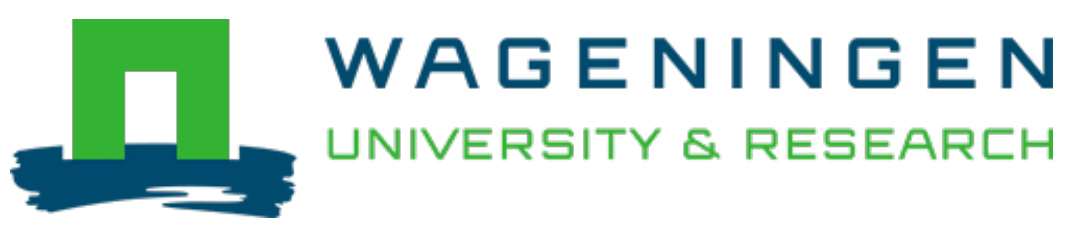

\title{
LEDs Make It Resilient: Effects on Plant Growth and Defense
}

Trends in Plant Science

Lazzarin, M.; Meisenburg, M.; Meijer, Davy; Ieperen, W.; Marcelis, L.F.M. et al https://doi.org/10.1016/j.tplants.2020.11.013

This article is made publicly available in the institutional repository of Wageningen University and Research, under the terms of article $25 \mathrm{fa}$ of the Dutch Copyright Act, also known as the Amendment Taverne. This has been done with explicit consent by the author.

Article $25 \mathrm{fa}$ states that the author of a short scientific work funded either wholly or partially by Dutch public funds is entitled to make that work publicly available for no consideration following a reasonable period of time after the work was first published, provided that clear reference is made to the source of the first publication of the work.

This publication is distributed under The Association of Universities in the Netherlands (VSNU) 'Article $25 \mathrm{fa}$ implementation' project. In this project research outputs of researchers employed by Dutch Universities that comply with the legal requirements of Article $25 \mathrm{fa}$ of the Dutch Copyright Act are distributed online and free of cost or other barriers in institutional repositories. Research outputs are distributed six months after their first online publication in the original published version and with proper attribution to the source of the original publication.

You are permitted to download and use the publication for personal purposes. All rights remain with the author(s) and / or copyright owner(s) of this work. Any use of the publication or parts of it other than authorised under article $25 \mathrm{fa}$ of the Dutch Copyright act is prohibited. Wageningen University \& Research and the author(s) of this publication shall not be held responsible or liable for any damages resulting from your (re)use of this publication.

For questions regarding the public availability of this article please contact openscience.library@,wur.nl 


\title{
Trends in
}

\section{Plant Science}

\section{LEDs Make It Resilient: Effects on Plant Growth and Defense}

\author{
M. Lazzarin, ${ }^{1,4}$ M. Meisenburg, ${ }^{2,4}$ D. Meijer, ${ }^{3,4}$ W. van leperen, ${ }^{1}$ L.F.M. Marcelis, ${ }^{1}$ I.F. Kappers, ${ }^{2}$ \\ A.R. van der Krol, ${ }^{2}$ J.J.A. van Loon, ${ }^{3}$ and M. Dicke ${ }^{3, *}$
}

Light spectral composition influences plant growth and metabolism, and has important consequences for interactions with plant-feeding arthropods and their natural enemies. In greenhouse horticulture, light spectral composition can be precisely manipulated by light-emitting diodes (LEDs), and LEDs are already used to optimize crop production and quality. However, because light quality also modulates plant secondary metabolism and defense, it is important to understand the underlying mechanisms in the context of the growth-defense trade-off. We review the effects of the spectral composition of supplemental light currently used, or potentially used, in greenhouse horticulture on the mechanisms underlying plant growth and defense. This information is important for exploring opportunities to optimize crop performance and pest management, and thus for developing resilient crop-production systems.

\section{Light Spectrum and Growth-Defense Trade-off}

Light is an important resource for plants that profoundly influences plant morphology, physiology and development. Changes in the spectral composition of light impact on processes ranging from photosynthesis to secondary metabolism [1,2]. In greenhouses, LEDs are increasingly used to exploit this knowledge and improve crop production and quality. LEDs provide possibilities to accurately control the spectral composition of supplemental light [3]. How different wavelengths of light influence plant growth, the photoreceptors and downstream responses that are involved, and what this implies for productivity and sustainability of greenhouse crop production have been extensively reviewed before [1-8]. However, although these reviews focus on commercial endpoints such as yield and quality, they often do not consider the effects on plant defenses. As predicted by the paradigm of the growth-defense trade-off, the use of LEDs to supplement solar light for the stimulation of growth also influences plant resistance to biotic stress $[9,10]$. For instance, in arabidopsis (Arabidopsis thaliana), low red:far-red (R:FR) ratios stimulate plant growth but reduce resistance to the plant pathogenic fungus Botrytis cinerea [11]. Key steps in growth and defense signaling pathways are responsive to light spectral composition [12-14]. Because of the growth-defense trade-off, a proper understanding of how LEDs influence plant physiology, including resistance to biotic stress, will be necessary to permit optimal application of LED lights in resilient greenhouse crop production.

Although light is an inevitable requirement for plant growth, excess light can cause photodamage and reduce crop productivity. The degree of excess illumination during photosynthesis depends on the wavelength [15]. Light spectral composition influences the concentration of foliar pigments and photoprotective compounds [16] that are involved in protection against both light stress and biotic stress. Balancing the effects of light spectrum on crop growth and crop protection is therefore required for optimal crop production in greenhouses. We address fundamental aspects of plant responses to supplemental light of specific wavelengths from the perspective of developing

\section{Highlights}

Light spectral composition influences plant morphology, physiology, and development by impacting on processes ranging from photosynthesis to secondary metabolism.

Signaling pathways underlying growth and defense are linked, and light spectral composition therefore influences both processes.

Light spectral composition influences the behavior of plant-feeding arthropods and their carnivorous enemies directly or via changes in plant morphology and physiology.

Specific manipulation of light spectral composition in greenhouses through LEDs may optimize both crop production and crop protection, thus improving crop resilience.

${ }^{1}$ Horticulture and Product Physiology, Wageningen University, PO Box 16, 6700 AA Wageningen, The Netherlands ${ }^{2}$ Laboratory of Plant Physiology, Wageningen University, PO Box 16 , 6700 AA Wageningen, The Netherlands ${ }^{3}$ Laboratory of Entomology, Wageningen University, PO Box 16, 6700 AA Wageningen, The Netherlands ${ }^{4}$ These authors contributed equally to this article

${ }^{*}$ Correspondence:

marcel.dicke@wur.nl (M. Dicke). 
resilient greenhouse crop production. We focus on herbivorous arthropods as biotic stress because their behavior, as well as the behavior of their natural enemies, may also be affected by supplemental light. We discuss the effects of adding far-red (FR, see Glossary), red (R), blue (B) or UV components of the light spectrum to ambient greenhouse conditions in three sections: (i) photosynthesis and plant growth, (ii) plant defense against arthropod herbivores, and (iii) plantarthropod interactions and arthropod behavior. Although we focus on the effects of supplemental LED lights of specific wavelengths, we note that similar results may be obtained with other artificial light sources that also provide specific wavelengths.

\section{Photosynthesis and Plant Growth}

Photosynthesis is the basis for plant growth. The spectral composition of light is an important determinant of light absorption and $\mathrm{CO}_{2}$ fixation efficiency [15], and induces a variety of morphological responses that optimize light-capturing ability [17]. The extent to which light quality can influence growth by changes in photosynthesis and light capture ability depends on crop developmental stage. Morphological adaptations induced by light spectrum are particularly important for light interception in young plants with a low leaf area index $(<3)$. Such effects in young plants may overrule the effects of light quality on photosynthesis at the leaf level [18]. In taller crops, the lower leaves may be exposed to fluctuating light in the form of sunflecks ( $<8 \mathrm{~min}$ and peak irradiance lower than above-canopy irradiance), sun patches ( $>8 \mathrm{~min}$ ) and sun gaps (>60 min), each of which may impose light stress on photosynthesis [19].

Light spectral composition can affect the ability of a plant to cope with light stress by influencing the biosynthesis of secondary metabolites that function as antioxidants [16]. Increasing the fraction of B light promotes photosynthesis and leaf thickness in cucumber and tomato [20,21] and stimulates stomatal opening in multiple plant species [22]. B light also increases the concentrations of leaf pigments such as chlorophylls and carotenoids in mustard, parsley, and beet [23], and enhances anthocyanin accumulation in lettuce [24]. By contrast, white light $(W)$ increases carotenoid levels more than either B or R light in red pak choi [25]. Under high levels of photosynthetically active radiation, UV-B light also increases net plant photosynthesis in several plant species, but excess UV-B can cause photodamage and the production of photoprotective pigments, and thereby reduces photosynthetic efficiency.

Low R:FR conditions may reduce the UV-B-mediated induction of plant flavonoids [26]. Acclimation to low R:FR results in reduced chlorophyll and carotenoid content in lettuce and tomato $[27,28]$ and in reduced levels of ascorbate and glutathione in common bean [29]. The reduced partitioning of dry matter to leaves coincides with a decrease in defense against abiotic and biotic stresses. How changes in light spectral composition influence photosynthesis and photoprotection in greenhouses, and how the light-mediated production of flavonoids influences defense against biotic stress, need further investigation.

\section{'Photoprotected' Phenotype}

Excess light may damage plant photosystems, and plants have several layers of protection to avoid such damage (Box 1). Not all energy that is absorbed by the antenna complex of photosystem II (PSII) is used to drive photochemistry, and the remaining energy must be dissipated as heat. Photoprotective mechanisms dissipate excess light through non-photochemical quenching (NPQ) which affects the production of pigments $[30,31]$ that scavenge reactive oxygen species (ROS), thus hampering growth [31-33]. Such ROS scavenging has consequences for plant defense because ROS accumulation can enhance defense against arthropod herbivores [34,35]. For example, npq4-1 mutant arabidopsis plants are more resistant to herbivorous spider mites than are wild-type plants [34].

\section{Glossary}

Blue (B) light: photons of wavelengths in the range 400-500 $\mathrm{nm}$. Cyclic electron flow around PSI (CEF-PSI): a series of alternative pathways in which electrons are excited by light at the primary chlorophyll electron donor within photosystem I (PSI, P700) and are then transferred through a series of redox carriers back to $P 700$.

Damage-associated molecular patterns (DAMPs): plant-derived compounds that originate from mechanical damage that activates plant responses.

Diapause: a form of developmental arrest in arthropods that is expressed in response to specific environmental conditions; diapause is a speciesspecific developmental stage that is not necessarily the stage exposed to the inducing conditions.

Far-red (FR) light: photons of wavelengths in the range $700-800 \mathrm{~nm}$. Green (G) light: photons of wavelengths in the range $500-565 \mathrm{~nm}$. Herbivory-associated molecular patterns (HAMPs): herbivore-derived compounds that activate plant responses.

Herbivory-induced plant volatiles (HIPVs): feeding by herbivorous arthropods results in the activation of the biosynthesis of plant volatiles, resulting in blends of herbivoryinduced volatiles.

Non-photochemical quenching (NPQ): a measure of the light (excitation) energy absorbed by the plant that is dissipated as heat. The dissipation decreases the amount of excitation energy reaching the photochemical reaction centers, particularly PSII, thus preventing photodamage. Photosystem I (PSI): the thylakoidmembrane protein complex that converts light energy to produce $\mathrm{NADPH}$ via electron transfer from plastocyanin to ferredoxin.

Photosystem II (PSII): the first protein complex in the light-dependent reactions of oxygenic photosynthesis; PSII is also located in the thylakoid membrane.

Phototaxis: movement towards or away from a light stimulus.

PsbS: a protein belonging to the chlorophyll a/b/xanthophyll-binding proteins (light-harvesting complex, LHC) superfamily that is involved in the regulation of NPQ and acts in the chloroplast as a lumen $\mathrm{pH}$ sensor. 


\section{Box 1. Photosynthesis and Photoprotection}

Upon exposure to light, photosynthesis converts light energy into chemical energy by synthesizing carbohydrates from $\mathrm{CO}_{2}$ and water. The absorption of light by light-harvesting complexes (LHCs) beyond what can be used by photochemistry can lead to harmful side reactions, collectively termed photoinhibition [115]. Plants have developed a range of photoprotection mechanisms to avoid and repair damage caused by excess light [116]. Non-photosynthetic pigments screen the sensitive photosystems from excess light at wavelengths $\leq 520 \mathrm{~nm}$ [15]. Once light is absorbed by photosynthetic pigments, dissipation of excess excitation energy as heat by non-photochemical quenching (NPQ) is an important protection mechanism [115]. When overexcitation of PSII is not accompanied by sufficient NPQ, excess energy causes the production of superoxide, singlet oxygen, and peroxide (ROS) that are particularly harmful to the oxygen-evolving complex of PSII. A variety of antioxidants eliminate these reactive photoproducts. Carotenoids exert their photoprotective action by rapidly quenching the excited states of the chlorophylls. Ascorbate protects against photoinhibition via the water-water cycle [31]. If all lines of protection fail, ROS can damage the D1 protein of PSII, which, if not sufficiently balanced by repair processes [116], eventually results in cell death [117]. In parallel, oxidative stress influences the cellular antioxidant network, in turn affecting plant growth [31] and defense against biotic stress [34,35].

Activation of NPQ requires the generation of a pH gradient across the thylakoid membrane (between stroma and lumen). The proton gradient induces the fast NPQ response (qE) through the conversion of the xanthophyll pigment violaxanthin (V) into zeaxanthin (Z) and the protonation of a peripheral antenna complex of PSII, PsbS. The slow relaxation kinetics of qE after a period of excess light delay reopening of PSII reaction centers, and restore the associated $\mathrm{CO}_{2}$ fixation rate and potentially growth [118]. In addition, NPQ modulation is achieved by altering the kinetic properties of the ATP synthase, which then controls the conductivity of the thylakoid membrane to protons, and therefore the pH gradient [115]. PSI has an additional photoprotective mechanism that involves cyclic electron flow around PSI (CEF-PSI) [37].

Other types of photoprotective mechanisms are associated with more long-lasting forms of 'unbalance' or 'stress'. They involve state transitions, the translocation of mobile LHCII between PSI and PSII (minutes) [38], and changes in the stoichiometry of the PSI/PSII ratio (days) [39].

Photoprotection may be manipulated by specific wavelengths to induce a 'photo-protected' phenotype (Figure 1). FR light enhances NPQ relaxation kinetics by preferentially exciting photosystem I (PSI) [36], thereby enhancing cyclic electron flow around PSI (CEF-PSI) in the high-light phase of fluctuating light [37] and during state transitions [38] (Box 1). In the short term, supplemental FR light may thus alleviate fluctuating-light stress by increasing photosynthetic efficiency and reducing the need to dissipate excess absorbed energy at PSII. In the long term, FR light induces adjustments in the stoichiometry of PSI:PSII to better balance photosystem excitation (low PSI:PSII ratio) [39]. Supplemental FR light also stimulates stomatal closure by influencing the plastoquinone redox state in guard cells [40], and thus influences the efficiency of $\mathrm{CO}_{2}$ fixation.

\section{'Shade-Avoidance' Phenotype}

A low R:FR ratio induces a range of 'shade-avoidance' responses (Figure 1C) which include leaf expansion, stem length, and upward leaf movement (hyponasty) [17,41]. The advantage of a 'shade-avoidance' phenotype is the enhanced ability to capture light, which is particularly important in a young crop.

In young plants, FR light enhances light interception through increased leaf area and petiole elongation [28]. In the fruiting stage, supplemental FR light may increase dry matter partitioning into the fruits and stem [42]. In addition, B light depletion and a relative increase in green (G) light are other characteristics of canopy shade that also trigger shade-avoidance responses in many plant species (Figure 1) $[17,43]$. Similarly to the production of photoprotective pigments, low R: FR and UV-B have contrasting effects, and UV-B can block the growth-inducing effects of low R:FR ratio $[26,44,45]$.

In conclusion, modulation of light spectrum by LEDs may influence changes in local resource partitioning, which causes configurational conflicts between the 'shade-avoidance' and
qE: the energy-dependent component of non-photochemical quenching (NPQ). Reactive oxygen species (ROS): unstable and reactive molecules with an oxygen group; ROS can damage DNA, RNA, and proteins.

Red (R) light: photons of wavelengths in the range 600-700 $\mathrm{nm}$.

UV light: wavelengths in the range 10-400 nm, commonly referred to UV-A (315-400 nm), UV-B (280-315 nm), and UV-C (100-280 nm).

Water-water cycle: in chloroplasts, this is the photoreduction of dioxygen to water in PSI by electrons generated in PSII from water.

White light (W): a combination of wavelengths, usually in the range 380-760 nm; may also refer to sunlight that has a wider range of wavelengths. 


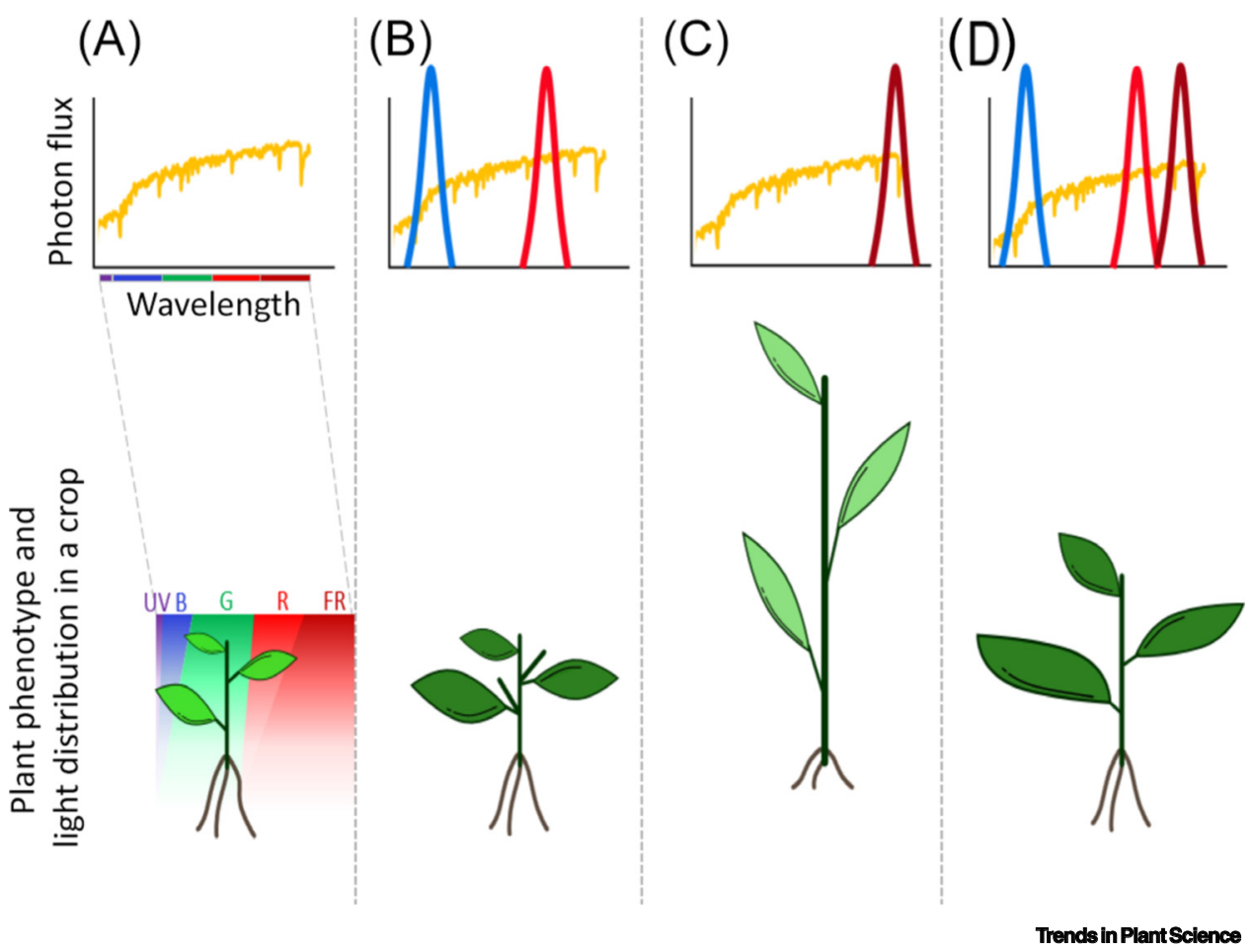

Figure 1. Effects of Light Color on Plant Phenotypic Traits. (A) Solar light drives photosynthesis and promotes light interception through changes in morphology. A vertical light intensity and spectrum gradient exists within the crop: red (R) and blue (B) light are mainly absorbed in the upper layers of the crop, whereas the absorption of far-red (FR) and green (G) as a fraction of total light increases from the top to the bottom layers of the canopy. The change in light spectrum from top to bottom of the canopy is indicated in the lower part of this panel. Panel (B) LEDs increase crop photosynthesis and growth. Supplemental B light as well as R plus B light increase leaf photosynthetic capacity by increased chlorophyll content (indicated by dark-green foliage) and leaf thickness [20], but can reduce apical dominance (i.e., increased formation of axillary buds) and induce self-shading [108,109]. In addition, it may induce 'photoprotected' phenotypes by increasing leaf concentrations of anthocyanins and carotenoids [23-25,27,30]. (C) Supplemental FR light induces the 'shade-avoidance' phenotype: slender shoots, rapid rates of stem or leaf elongation, and high apical dominance, allowing leaves to increase their exposure to sunlight [46]. This is typically coupled to low leaf mass per area and reduced allocation to roots [28]. (D) Supplemental R light and B light in combination with FR light can increase leaf photosynthesis and light interception ability through leaf area expansion, whereas a more upright leaf angle promotes deeper light penetration in the crop.

'photo-protected' phenotypes [46], and, depending on the crop developmental stage, can enhance either plant growth or resilience.

\section{Plant Defense Against Arthropod Herbivores}

When plants exhibit enhanced growth, defensive capabilities decrease, and vice versa [9]. Plant defenses are regulated by light modulation of endogenous phytohormonal signal-transduction pathways [47]. An important component of plant defense consists of inducible defenses that are activated by arthropod feeding and are orchestrated by the salicylic acid (SA) and jasmonic acid (JA) signaling pathways (Box 2) [48]. Infection by biotrophic pathogens (e.g., powdery mildew) or phloem-feeding arthropods (e.g., aphids and whiteflies) locally induces ROS production, resulting in SA biosynthesis and leading to a local hypersensitive response and systemic acquired resistance. Activation of the JA signaling pathway by leaf-chewing herbivores such as caterpillars and beetles leads to the formation of defense-related secondary metabolites. The SA and JA pathways interact antagonistically (Figure 2) [48]. Some phloem-feeding arthropods exploit this antagonism to trick plants into an SA response to suppress the JA response [49]. 


\section{Box 2. Phytohormones in Plant Defense}

Plants perceive biotic attack via various cues, and respond by activating specific defenses. Different herbivore feeding guilds are recognized through damage-associated molecular patterns (DAMPs) and herbivory-associated molecular patterns (HAMPs) that activate different phytohormonal signaling pathways [119]. Wounding induced by chewing arthropods such as caterpillars predominantly results in the biosynthesis of jasmonic acid (JA) and abscisic acid (ABA) [49,82]. By contrast, phloem feeders such as aphids predominantly induce the synthesis of salicylic acid (SA) $[55,82]$. Both herbivore feeding guilds may also induce ethylene (ET) [49].

JA-lle

$\mathrm{JA}$ is converted into the bioactive compound JA-isoleucine (JA-lle). In the absence of JA-lle, a repressor complex consisting of JASMONATE ZIM-DOMAIN (JAZ), NINJA, and TOPLESS blocks the transcriptional activity of JASMONATE INSENSITIVE 2/3/4 (MYC2/3/4) transcription factors (TFs) [12,82]. Upon herbivory, JA-lle binds to CORONATINE INSENSITIVE 1 (COI1) of the E3 ubiquitin ligase complex SCF ${ }^{\mathrm{CO}}{ }^{1}$, resulting in degradation of JAZ proteins and transcription activation by MYC TFs of target genes such as VEGETATINE STORAGE PROTEIN 1 (VSP1), which has antiherbivore activity, and by other MYB TFS that are involved in the biosynthesis of defense-related secondary metabolites, that act as deterrents or toxins to arthropod herbivores, and of volatiles that are involved in attracting enemies of the arthropods [82].

\section{JA-lle/ABA}

ABA is usually induced through abiotic stresses, such as drought or salinity, but can also be upregulated during herbivory [49]. The main effect of ABA in defense is in downregulating the transcription factors ETHYLENE RESPONSE FACTOR 1 (ERF1)/OCTADECANOID-RESPONSIVE ARABIDOPSIS (ORA59), and consequently downregulating the defense gene PLANT DEFENSIN 1.2 (PDF1.2).

\section{JA-lle/ET}

In the absence of ET, the TF ETHYLENE INSENSITIVE 3 (EIN3)/ETHYLENE-INSENSITIVE3-LIKE 1 (EIL1) is targeted for degradation by the E3 ubiquitin ligase complex containing EIN3-BINDING F BOX PROTEIN 1/2 (EBF1/2) [49]. ET results in cleavage of ETHYLENE INSENSITIVE 2 (EIN2) at the endoplasmic reticulum membrane, and the released C-terminal part of EIN2 moves to the nucleus to prevent interaction of EIN3/EIL1 with EBF1/2. Stabilized EIN2/EIL1 subsequently activates the ethylene-responsive TF ERF1. ERF1 activates TF ORA59 which subsequently activates transcription of PDF1.2 as part of the hypersensitive response.

SA

SA production is triggered by ROS [55,60,82], and SA in turn enhances ROS production. In the cytosol, ROS/SA reduces a complex consisting of NONEXPRESSOR OF PR1 (NPR1) oligomers to monomer NPR1. Monomeric NPR1 can enter the nucleus and activate the TGA TF bound to different target promoters. This leads to the activation of pathogenesisrelated (PR) genes and WRKY TF-responsive downstream genes that are involved in hypersensitive responses or systemic acquired resistance.

Many fundamental studies show the involvement of specific narrow-bandwidth light signaling in defense responses (Figure 2).

\section{Effects of FR Light on Phytohormone-Mediated Defense}

JA is converted into the biologically active JA-isoleucine (JA-lle) by JASMONATE RESISTANT 1 (JAR1/FIN219) [50]. FR light influences JA-lle signaling in plants: intrinsic JA levels are reduced by the activity of a FR-induced sulfotransferase [54], and JAR1 protein levels are reduced under FR light [51]. However, at the same time, FR light enhances the intrinsic activity of JAR1 proteins through the FR light-inducible FIN219-INTERACTING PROTEIN 1 (FIP1) (Figure 2) [50]. Under supplemental FR light JA-lle signaling contributes to the suppression of the shadeavoidance elongation response [51]. To understand how the effects of FR light on JA-lle signaling influence plant defense against arthropod herbivory, the effects of FR light should be studied for plants under herbivore attack.

Downstream of JA-lle signaling are the JASMONATE-INSENSITIVE (MYC) transcription factors, which are suppressed by the JASMONATE ZIM-DOMAIN (JAZ) protein complexes in the absence of JA-lle (Figure 2 and Box 2). JA-lle triggers degradation of the JAZ proteins, thus releasing MYC transcriptional activity [13]. The protein stability of MYCs and JAZs is regulated 


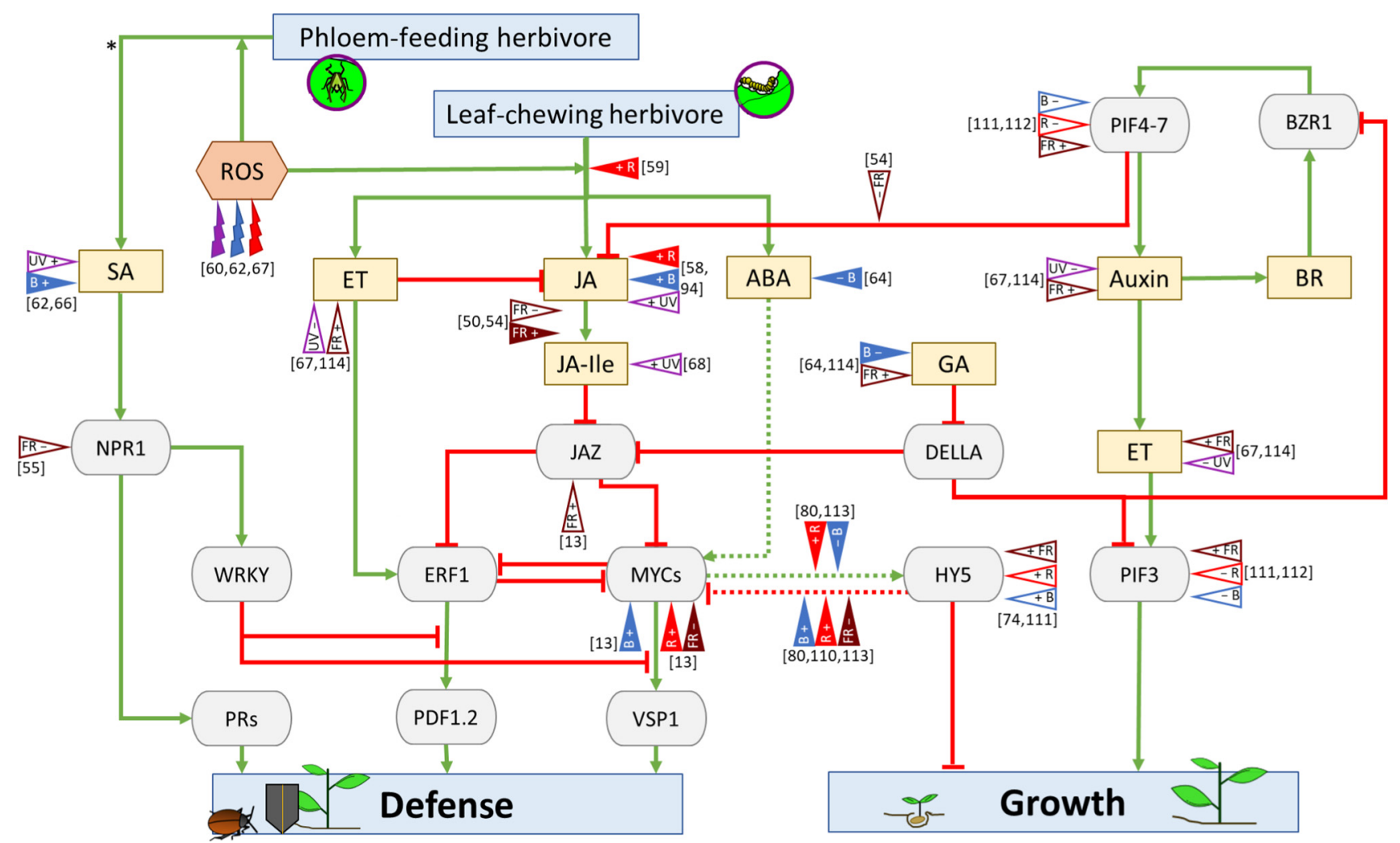

Figure 2. Effects of Light Color on Signaling Pathways Underlying Plant Growth and Defense. Green arrows indicate positive regulation, and red blunt-end lines indicate negative regulation. Dotted lines: interaction requires that MYC transcription factor is released from its repressor complex by isoleucine-coupled jasmonic acid (JA-lle) signaling. Unicolored triangles indicate the regulation of gene or protein activity by monochromatic light; triangles with a colored border show regulation by a supplemental light spectrum. Blue triangles indicate blue (B) light-mediated regulation via cryptochromes and phototropins; bright-red triangles indicate regulation by red (R) light; dark red triangles indicate regulation by far-red (FR) light (or both R and FR) light that is mediated via phytochromes. Symbols: + and - in triangles indicate positive and negative regulation, respectively; ${ }^{*}$, induction of the SA pathway by phloem-feeding arthropods is considered to be a way to interfere with the more effective JA pathway. Compounds (indicated in hexagons): ROS, reactive oxygen species. Hormones (indicated in squares): ABA, abscisic acid; BR, brassinosteroids; ET, ethylene; GA, gibberellic acid (GA); JA, jasmonic acid; JA-lle, JA-isoleucine; SA, salicylic acid. Genes and transcription factors (indicated in ovals): BZR1, BRASSINOSTEROIDS RESISTANT 1 (BZR1); DELLA, DELLA proteins; ERF1, ETHYLENE RESPONSE FACTOR 1; HY5, ELONGATED HYPOCOTYL 5; JAZ, JASMONATE ZIM-DOMAIN; MYCS, JASMONATE INSENSITIVE; NPR1, NONEXPRESSOR OF PR1; PDF1.2, PLANT DEFENSIN 1.2; PIF3-7, PHYTOCHROME INTERACTING FACTORS 3-7; PRs, PATHOGEN RESISTANCE genes; VSP1, VEGETATIVE STORAGE PROTEIN 1; WRKY, WRKY DNA-BINDING protein. NUmbers in brackets refer to corresponding references. Additional growth-related references are given in [110-114].

by R:FR, and FR light has a destabilizing effect on MYCs and a stabilizing effect on JAZ proteins [13]. The stabilizing effect of FR on JAZ proteins results from two mechanisms: (i) shade conditions lead to an increase in gibberellin signaling, which targets DELLA proteins for degradation [52]. Because DELLAs antagonize the action of JAZ proteins, shade could thus result in higher JAZ protein repressor activity [52,53]. (ii) FR light stabilizes PIF transcription factors that induce the expression of a gene encoding JA sulfotransferase, resulting in lower levels of JA-lle under shade conditions [54] (Figure 2).

Changes in R:FR ratio are sensed by the photoreceptor PHYTOCHROME B (PHYB), and FR light reduces PHYB signaling. Although SA levels are reduced in the arabidopsis phyB mutant, a reduction in PHYB signaling by shade or FR light does not change basal (non-induced) SA levels in wild-type plants $[55,56]$. However, shade-grown plants are more vulnerable to SA-inducing organisms, and this may be explained by repression of downstream SA signaling through 
inhibition of nuclear transport of NONEXPRESSOR OF PR1 (NPR1), the main regulator of SA signaling (Figure 2) $[44,55]$. Translocation of NPR1 into the nucleus requires phosphorylation, and FR light inhibits the required kinase activity [55]. Indeed, the NPR1 target gene PATHOGEN RESISTANCE 1 (PR1), a marker gene of SA-regulated defense, is downregulated in shade-grown plants [57].

\section{Effects of B Light on Phytohormone-Mediated Defense}

Basal JA levels are affected by light spectral composition, and the combination of $\mathrm{R}$ and $\mathrm{B}$ light results in higher JA levels compared to W light [58]. However, herbivory under W light caused a sharp increase in JA levels, but had little effect on the JA levels under R and B light $[58,59]$. MYC proteins are stabilized under $R$ and $B$ light [13]. However, it remains to be investigated how this influences defense capacity.

B light can lead to ROS production in plants, leading to enhanced basal SA levels [60-62] and enhanced defense against biotrophic pathogens, while activating transcriptional suppressors (WRKYs) of downstream JA-dependent defense genes [63] (Figure 2). By contrast, B light leads to reduced levels of abscisic acid (ABA) and gibberellic acid (GA) (Box 2) [64,65]. B light reduces GA levels, resulting in stabilization of DELLA proteins and subsequently destabilization of JAZ [65]. Thus, B light may lead to derepression of JA-regulated defense (Figure 2). More research will be necessary to determine how the intensity, timing, and duration of supplemental $B$ light influences these dual effects of $B$ light on defense signaling so as to determine the most effective stimulation of either SA- or JA-mediated defense.

Effects of UV-B on Phytohormone-Mediated Defense

Most natural UV light does not penetrate the glass of greenhouses, but may be supplied by UV LEDs. Even though UV-B can be harmful to plants as a result to DNA damage and ROS production, small doses of UV-B can improve plant defense [66-69]. Multiple studies show that low doses of UV-B light increase both basal JA and SA levels as well as the basal expression level of several defense-related genes [70]. Indeed, there is a large overlap between transcriptional responses to UV-B-, SA-, JA-, and herbivory-treated plants [70]. Similarly, short exposure to UV increases the resistance of tomato to western flower thrips (Frankliniella occidentalis) via increased JA and SA levels and elevated expression of defense-related genes such as THREONINE DEAMINASE (TD), JASMONIC-INDUCED PROTEIN 21 (JIP-21), and PATHOGENESIS-RELATED GENE 6 (PR6) in tomato leaves [68]. Similar results were found in a field trial with soybean plants [66], indicating the possible application of UV LEDs for pest management.

\section{Light Spectrum and Defense Metabolites}

Defense compounds may be produced constitutively or after induction by herbivore feeding. Many defense-related metabolites provide added nutritional value to crops, and a wealth of information on the effects of light spectral composition on crop quality is available [41,71-79]. Only a few studies have linked these effects to actual defense against arthropods [75,80]. For example, flavonoids protect plants against insects by influencing the behavior, growth, and development of arthropods [80]. As discussed earlier, flavonoids are also important for photoprotection, and their biosynthesis can be enhanced through the addition of $\mathrm{R}$ and $\mathrm{B}$ light but reduced by $\mathrm{FR}$ light.

In addition to direct defenses against herbivorous arthropods, plants have indirect defenses involving the release of herbivore-induced plant volatiles (HIPVs). HIPVs include volatile fatty acid-derived compounds, terpenoids, and phenylpropanoids/benzenoids that attract the enemies of herbivorous arthropods [81]. Light spectral composition may affect volatile quantities, and $R$ and $B$ light result in increased emissions [79], whereas FR light decreases total emissions 
[71-73]. However, light spectral composition may also affect the quantitative composition of the volatile blend. For instance, FR light reduces the emission of the homoterpene $(E, E)-4,8,12-$ trimethyl-1,3,7,11-tridecatetraene that is involved in attracting natural enemies of herbivores [82], whereas the emission of other volatiles was unaffected. Over longer time-periods, FR light may also reduce the number of glandular trichomes, which are essential for releasing volatiles in plant species such as tomato [71]. The underlying mechanisms of how light spectrum modulates the biosynthesis and emission of volatiles needs further elucidation to be able to assess the consequences of light modulation on indirect plant defense.

\section{Plant-Arthropod Interactions and Arthropod Behavior}

Similarly to plants, most arthropods are phototactic organisms that possess a range of photoreceptors sensitive to a selection of wavelengths, and which influence different aspects of arthropod behavior such as host finding and navigation [83]. Changing light spectral composition to manipulate plant development may therefore influence the behavior, distribution, and population development of arthropods in greenhouses. Furthermore, the changes in plant morphology and physiology induced by supplemental light, as described above, may influence plant-arthropod interactions via changes in direct and indirect plant defense traits [71]. Distinguishing between direct and plant-mediated effects of supplemental light on arthropod behavior is crucial for effective pest management in greenhouses.

\section{Effects of Light Spectrum on Herbivore Behavior}

Most herbivorous arthropods possess a trichromatic visual system that has peak sensitivities in the UV, B, and G parts of the spectrum [84]. Using LEDs, the spectral sensitivity of major agricultural pests is being elucidated in more detail [83,85]. Understanding arthropod phototaxis to particular wavelengths can be important for implementing LED-based improvement of pest management [84], especially for the development of light traps [86]. However, the most efficient colors for attracting arthropods (green/yellow) often have little benefits for crop production.

Wavelength-dependent behavior of arthropods can also be dependent on color contrasts rather than on single wavelengths. Instead of attracting pests towards a light source for trapping, some wavelengths may also be applied to repel herbivorous arthropods. Several hemipteran species exhibit a mechanism of B-G opponency in which higher proportions of B light cause repellence, and these insects use this contrast as the main determinant for host acceptance or settling behavior [83,87]. The addition of $\mathrm{R}$ light reduces plant attractiveness to melon thrips [88], possibly through reduced perception of 'greenness' of plants under supplemental $R$ light. These studies indicate that the supplementation of $\mathrm{R}$ and $\mathrm{B}$ light in greenhouses might be effective in reducing plant attractiveness to herbivorous pests, while at the same time contributing to crop production.

Light spectral composition can also directly influence the life-history traits of arthropods. For example, B light inhibits diapause induction in herbivorous mites [89]. However, evidence for direct effects of light spectral composition on arthropod physiology is still scarce and requires further study.

Plant-Mediated Effects of Light Spectrum on Herbivorous Arthropods

Morphological changes in plants resulting from supplemental light may influence plant-arthropod interactions. For example, the density of glandular trichomes that provide resistance to arthropod herbivores [90] can be influenced by light spectral composition [71]. Changes in leaf thickness and 'greenness' in response to varying R:FR ratios altered the attractiveness of plants for whiteflies [91], indicating that leaf morphology and color can be important aspects of light-mediated plant-arthropod interactions [46]. 
Changes in plant defensive physiology resulting from supplemental LED light also have marked consequences for the performance and population dynamics of herbivorous arthropods. Importantly, downregulation of defenses in response to a low R:FR ratio may considerably increase herbivore performance and their feeding damage $[71,92]$. Such effects are dependent on the degree of host-plant specialization of the herbivorous arthropods. Although different supplemental wavelengths can change the metabolite profile of brassicaceous plants by increasing glucosinolate content, none of these changes affected the performance of the specialist cabbage aphid (Brevicoryne brassicae) [77,93]. UV-B increased the resistance of arabidopsis to generalist Spodoptera litura caterpillars because of increased levels of JA-regulated glucosinolates and other, unidentified, secondary metabolites [94]. Supplemental R light did not influence the performance of several generalist herbivores on tomato plants, but decreased the performance of a specialist caterpillar (D.M. et al., unpublished).

Supplemental light can also influence emissions of plant volatiles. Low R:FR light illumination reduces the production of constitutive plant volatiles, resulting in reduced attraction of specialist herbivores that use these volatiles for host finding [72]. Exposure to low R:FR ratios also influences the emission of some HIPVs in tomato plants, making them more attractive to the hemipteran biological control agent Macrolophus pygmaeus. These results indicate a shift from direct towards indirect defense when tomato plants are exposed to supplemental FR light [71].

\section{Direct and Indirect Effects of Light Spectrum on Beneficial Arthropods}

The use of arthropods for biological control and pollination has become increasingly popular since the late 1900s [95,96], and has tremendously enhanced the sustainability of greenhouse crop production $[95,96]$. It is therefore also important to determine whether changes in light spectral composition influence the behavior and effectiveness of these beneficial arthropods.

Most parasitoids used for biological control belong to the Hymenoptera, which possess the same trichromatic visual system as many herbivorous arthropods [84,97]. Although research on the effects of light spectral composition on these natural enemies is still scarce, there are promising reports that biological control can be improved through changes in light spectral composition. For example, an increased proportion of B light affects the sex ratio of the aphid parasitoid Aphidius ervi, resulting in more females [98]. Moreover, extending the photoperiod with a mixture of $R$ and $B$ light increased parasitism rates on the pea aphid (Acyrthosiphon pisum) [98].

Most pollinators, including bumble bees that are extensively used to pollinate greenhouse crops such as tomato, also belong to the Hymenoptera and share the same trichromatic (UV, B, G) visual system [97], although some families have an additional R light receptor [99]. However, little is known about how supplemental light influences their behavior under greenhouse conditions. A recent study showed that combinations of $\mathrm{R}$ and $\mathrm{B}$ light did not affect the landing behavior of bumble bees compared to W light [100]. In this study, landing behavior was recorded on an artificial landing platform in the absence of flowers. Supplemental light, especially FR, is known to influence flowering traits [1]. How LED-induced changes in flower traits influence plant-pollinator interactions remains to be studied.

Whether or how light quality can be used to promote pest management may depend on the pest species occurring in the greenhouse because specialist and generalist herbivores respond differently to plant defensive chemistry. Moreover, changes in light spectral composition have different effects on herbivores that use either visual or volatile cues for host-plant selection. Differences in peak spectral sensitivity between herbivores and their natural enemies, that share the same trichromatic visual system, may be exploited in the development of effective light traps [101]. 
In conclusion, supplemental LED lights can influence beneficial and pest arthropods directly or via changes in plant traits. Unraveling which wavelengths hamper pest arthropods and enhance the effects of beneficial arthropods will provide opportunities to enhance pest management and contribute to resilient crop production. More studies will be necessary to unravel whether supplemental LED lights can be found that directly hamper pest arthropods and enhance the effects of beneficial arthropods, while at the same time having beneficial effects on growth and/or (in)direct plant defenses.

\section{Concluding Remarks and Future Perspectives}

Extensive studies on the effects of narrow-bandwidth light have resulted in detailed knowledge of the effects of R, FR, B, and UV-B light on plant growth and development. In addition, recent studies have also revealed that light spectrum may affect plant defense against herbivorous arthropods. Nevertheless, implementation of LEDs in greenhouse horticulture has mostly focused on energy-efficient ways to promote crop yield and product quality, whereas the consequences for pest management are less well explored [102]. LEDs may be used to precisely modulate spectral composition in time and space.

\section{Timing of Supplemental LED Light}

Although the use of supplemental LED light to support crop growth mostly involves continuous light treatments from a fixed position, initial studies on varying the timing and location of supplemental lights suggest that more refined light treatments may be able to circumvent the trade-off between growth and development. More research to assess the effects of timing and provision of short pulses of supplemental light is needed [103]. Although some knowledge of the effects of light pulses on plant growth is already available (e.g., effects of end-of-day FR treatments on flowering and quality of ornamental crops) [104], the consequences for host-plant resistance and pest management need further investigation (see Outstanding Questions).

\section{Location of Supplemental LED Light}

Effective steering of growth and defense may be especially possible at positions or times where natural radiation is limiting. For instance, even when high light intensity is available at the top of the canopy at noon, light levels at the bottom of the canopy may be low, and local manipulation of the light spectrum may be achieved there. The lack of FR in supplemental light sources negatively influences canopy photosynthesis [105]. Supplemental FR light could be used to increase photosynthesis in the lower canopy, provided that the effect on growth is not offset by a reduction in defense. Establishing such applications requires investigation of the underlying mechanisms and their effects under greenhouse conditions.

\section{Effect of Supplemental LED Light on Arthropods}

First studies show that supplemental LED light influences the performance of arthropods, including pollinators, pests, and their natural enemies. These insights warrant further studies on such effects of LED lights to avoid negative consequences. The focus should be on developing applications of LED lights that promote the effectiveness of beneficial arthropods while reducing the performance of pest arthropods. Greenhouse horticulture extensively uses integrated pest management (IPM) of which the main components are host-plant resistance and biological control to manage arthropod pests $[96,106]$. IPM in greenhouses has been carefully developed based on understanding the underlying mechanisms and ecology. Moreover, an important component is the use of bumblebees for pollination of crops such as tomato [107]. To integrate the use of LED lights to enhance yield and support IPM, a thorough understanding is needed of how supplemental LED lights simultaneously influence both plant (growth and defense) and arthropod pests, their natural enemies, as well as pollinators.

\section{Outstanding Questions}

Can the timing and duration of supplemental LED light of specific wavelengths contribute to simultaneous optimization of crop defense and growth? This requires better understanding of how supplemental light influences the different steps in the signaling pathways underlying plant defense and their effects on resistance to herbivorous arthropods.

What are the major positions of spectral perception and/or action in the plant? Can we use this knowledge to steer plant defense and growth by localized lighting in the canopy?

Does long-term acclimation to light spectrum influence instantaneous plant responses to light spectrum? Is there a memory effect after application of specific light spectra, and, if so, how long does it last?

Can supplemental UV and G light be used to direct growth and defense in greenhouse crops? How will this affect arthropod phototaxis and behavior?

Can supplemental LEDs be used to interfere with the suppression of JA responses by phloem-feeding arthropods in the greenhouse?

Because B light may influence both JA and SA signaling pathways, can the intensity and duration of supplemental $B$ light be used to selectively stimulate either SA- or JA-mediated defense?

How does supplemental light affect the biosynthesis of herbivore-induced plant volatiles and the relative strengths of direct and indirect plant defense against herbivorous arthropods?

What are the plant-mediated effects of supplemental light on the physiology and development of herbivorous arthropods? 
In conclusion, LEDs in greenhouses may provide specific additions to light spectral composition, timing, and location. To optimize the effects of LED lights, we need an integrated understanding of the fundamental mechanisms that underlie how light spectral composition influences crop growth and defense as well as the behavior of pest and beneficial arthropods. This involves the inclusion of temporal and spatial effects. If the addition of specific wavelengths to enhance crop growth or crop defense can be done at appropriate times and locations without interfering with the effectiveness of biological control agents or pollinators, then new approaches to implementation can be developed. A true understanding of how supplemental lighting by LEDs can be used to steer the dynamics between crop growth and defense will improve the resilience of greenhouse crop production.

\section{Acknowledgments}

Our research is funded by the Dutch Research Council (NWO, project number ALWGR.2017.004), with financial contributions by Biobest Biological Systems, Enza Seeds, Florensis, Schoneveld Breeding, WPK Vegetable Plants, and Heliospectra through the NWO.

\section{References}

1. Demotes-Mainard, S. et al. (2016) Plant responses to red and far-red lights, applications in horticulture. Environ. Exp. Bot $121,4-21$

2. Huché-Thélier, L. et al. (2016) Light signaling and plant responses to blue and UV radiations: perspectives for applications in horticulture. Environ. Exp. Bot. 121, 22-38

3. Dueck, T. et al. (2016) Light perception, signalling and plant responses to spectral quality and photoperiod in natural and horticultural environments. Environ. Exp. Bot. 121, 1-3

4. Davis, P.A. and Burns, C. (2016) Photobiology in protected horticulture. Food Energy Secur. 5, 223-238

5. Bantis, F. et al. (2018) Current status and recent achievements in the field of horticulture with the use of light-emitting diodes (LEDs). Sci. Hortic. 235, 437-451

6. Kusuma, P. et al. (2020) From physics to fixtures to food: current and potential LED efficacy. Hortic. Res. 7, 56

7. Yang, J. et al. (2019) Stomatal opening and growth in tomato seedlings treated with different proportions of red and blue light. Can. J. Plant Sci. 99, 688-700

8. de Wit, M. et al. (2016) Light-mediated hormonal regulation of plant growth and development. Annu. Rev. Plant Biol. 67, 513-537

9. Courbier, S. and Pierik, R. (2019) Canopy light quality modulates stress responses in plants. iScience 22, 441-452

10. Zust, T. and Agrawal, A.A. (2017) Trade-offs between plant growth and defense against insect herbivory: an emerging mechanistic synthesis. Annu. Rev. Plant Biol. 68, 513-534

11. Cargnel, M.D. et al. (2014) Linking phytochrome to plant immunity: low red:far-red ratios increase Arabidopsis susceptibility to Botrytis cinerea by reducing the biosynthesis of indolic glucosinolates and camalexin. New Phytol. 204, 342-354

12. Guo, Q. et al. (2018) JAZ repressors of metabolic defense promote growth and reproductive fitness in Arabidopsis. Proc. Natl. Acad. Sci. U. S. A. 115, E10768-E10777

13. Chico, J.M. et al. (2014) Repression of jasmonate-dependent defenses by shade involves differential regulation of protein stability of MYC transcription factors and their JAZ repressors in Arabidopsis. Plant Cell 26, 1967-1980

14. Riemann, M. et al. (2003) Impaired induction of the jasmonate pathway in the rice mutant hebiba. Plant Physiol. 133, 1820-1830

15. Hogewoning, S.W. et al. (2012) Photosynthetic quantum yield dynamics: from photosystems to leaves. Plant Cell 24, 1921-1935

16. Shimizu, H. (2016) Effect of light quality on secondary metabo lite production in leafy greens and seedlings. In LED Lighting for Urban Agriculture (Kozai, T. et al., eds), pp. 239-260, Springer Science+Business Media

17. Ballaré, C.L. and Pierik, R. (2017) The shade-avoidance syndrome: multiple signals and ecological consequences. Plant Cell Environ. 40, 2530-2543
18. Hogewoning, S.W. et al. (2010) An artificial solar spectrum substantially alters plant development compared with usual climate room irradiance spectra. J. Exp. Bot. 61, 1267-1276

19. Kaiser, E. et al. (2018) Fluctuating light takes crop photosynthesis on a rollercoaster ride. Plant Physiol. 176, 977-989

20. Hogewoning, S.W. et al. (2010) Blue light dose-responses of leaf photosynthesis, morphology, and chemical composition of Cucumis sativus grown under different combinations of red and blue light. J. Exp. Bot. 61, 3107-3117

21. Zhang, Y. et al. (2019) Red/blue light ratio strongly affects steady-state photosynthesis, but hardly affects photosynthetic induction in tomato (Solanum lycopersicum). Physiol. Plant. 167, 144-158

22. Inoue, S.I. and Kinoshita, T. (2017) Blue light regulation of stomatal opening and the plasma membrane H+-ATPase. Plant Physiol. 174, 531-538

23. Samuolienè, G. et al. (2017) Blue light dosage affects carotenoids and tocopherols in microgreens. Food Chem. 228, 50-56

24. Li, Q. and Kubota, C. (2009) Effects of supplemental light quality on growth and phytochemicals of baby leaf lettuce. Environ. Exp. Bot. 67, 59-64

25. Frede, K. et al. (2019) Light quality-induced changes of carotenoid composition in pak choi Brassica rapa ssp. chinensis. J. Photochem. Photobiol. B Biol. 193, 18-30

26. Escobar-Bravo, R. et al. (2017) Interactive effects of UV-B light with abiotic factors on plant growth and chemistry, and their consequences for defense against arthropod herbivores. Front. Plant Sci. 8, 278

27. Mickens, M.A. et al. (2018) A strategic approach for investigating light recipes for 'outredgeous' red romaine lettuce using white and monochromatic LEDs. Life Sci. Sp. Res. 19, 53-62

28. Kalaitzoglou, P. et al. (2019) Effects of continuous or end-ofday far-red light on tomato plant growth, morphology, light absorption, and fruit production. Front. Plant Sci. 10, 322

29. Bartoli, C.G. et al. (2009) Control of ascorbic acid synthesis and accumulation and glutathione by the incident light red/far red ratio in Phaseolus vulgaris leaves. FEBS Lett. $583,118-122$

30. Duan, L. et al. (2020) Red and blue light differentially impac retrograde signalling and photoprotection in rice. Philos. Trans. R. Soc. B Biol. Sci. 375, 20190402

31. Plumb, W. et al. (2018) Ascorbate-mediated regulation of growth, photoprotection, and photoinhibition in Arabidopsis thaliana. J. Exp. Bot. 69, 2823-2835

32. Külheim, C. et al. (2002) Rapid regulation of light harvesting and plant fitness in the field. Science 297, 91-93

33. Frenkel, M. et al. (2009) Improper excess light energy dissipation in Arabidopsis results in a metabolic reprogramming. BMC Plant Biol. 9, 12 
34. Barczak-Brzyżek, A.K. et al. (2017) Cross-talk between high light stress and plant defence to the two-spotted spider mite in Arabidopsis thaliana. Exp. Appl. Acarol. 73, 177-189

35. Jänkänpää, H.J. et al. (2013) Non-photochemical quenching capacity in Arabidopsis thaliana affects herbivore behaviour. PLoS One 8, e53232

36. Kono, M. et al. (2020) Far-red light accelerates photosynthesis in the low-light phases of fluctuating light. Plant Cell Physiol. 61 192-202

37. Kono, M. et al. (2017) Photoprotection of PSI by far-red light against the fluctuating light-induced photoinhibition in Arabidopsis thaliana and field-grown plants. Plant Cell Physiol. $58,35-45$

38. Taylor, C.R. et al. (2019) Demonstration of a relationship between state transitions and photosynthetic efficiency in a higher plant. Biochem. J. 476, 3295-3312

39. Wientjes, E. et al. (2017) Imaging the photosystem //photosystem chlorophyll ratio inside the leaf. Biochim. Biophys. Acta Bioenerg. 1858, 259-265

40. Busch, F.A. (2014) The red-light response of stomatal movement is sensed by the redox state of the photosynthetic electron transport chain. Photosynth. Res. 119, 131-140

41. Cerrudo, I. et al. (2017) Exploring growth-defence trade-offs in Arabidopsis: phytochrome B inactivation requires JAZ10 to suppress plant immunity but not to trigger shade-avoidance responses. Plant Cell Environ. 40, 635-644

42. Ji, Y. et al. (2019) Far-red radiation increases dry mass partitioning to fruits but reduces Botrytis cinerea resistance in tomato. Environ. Exp. Bot. 168, 103889

43. Ballaré, C.L. (1999) Keeping up with the neighbours: phytochrome sensing and other signalling mechanisms. Trends Plant Sci. 4, 97-102

44. de Wit, M. et al. (2013) Perception of low red:far-red ratio compromises both salicylic acid- and jasmonic acid-dependent pathogen defences in Arabidopsis. Plant J. 75, 90-103

45. Hayes, S. et al. (2014) UV-B detected by the UVR8 photoreceptor antagonizes auxin signaling and plant shade avoidance. Proc. Natl. Acad. Sci. U. S. A. 111, 11894-11899

46. Ballaré, C.L. and Austin, A.T. (2019) Recalculating growth and defense strategies under competition: key roles of photoreceptors and jasmonates. J. Exp. Bot. 70, 3425-3436

47. Moreno, J.E. and Ballaré, C.L. (2014) Phytochrome regulation of plant immunity in vegetation canopies. J. Chem. Ecol. 40, 848-857

48. Pieterse, C.M.J. et al. (2012) Hormonal modulation of plant mmunity. Annu. Rev. Cell Dev. Biol. 28, 489-521

49. Verma, V. et al. (2016) Plant hormone-mediated regulation of stress responses. BMC Plant Biol. 16, 86

50. Chen, C.Y. et al. (2017) Structural basis of jasmonate-amido synthetase FIN219 in complex with glutathione S-transferase FIP1 during the JA signal regulation. Proc. Natl. Acad. Sci. U. S. A. 114, E1815-E1824

51. Swain, S, et al, (2017) FAR-RED INSENSITIVE 219/JAR1 contributes to shade avoidance responses of Arabidopsis seedlings by modulating key shade signaling components. Front. Plant Sci. 8, 1901

52. Leone, M. et al. (2014) To grow or defend? Low red:far-red ratios reduce jasmonate sensitivity in Arabidopsis seedlings by promoting DELLA degradation and increasing JAZ10 stability. New Phytol. 204, 355-367

53. Djakovic-Petrovic, T. et al. (2007) DELLA protein function in growth responses to canopy signals. Plant J. 51, 117-126

54. Fernández-Milmanda, G.L. et al. (2020) A light-dependent molecular link between competition cues and defence responses in plants. Nat. Plants 6, 223-230

55. Nozue, K. et al. (2018) Network analysis reveals a role for salicylic acid pathway components in shade avoidance. Plant Physiol. 178, 1720-1732

56. Griebel, T. and Zeier, J. (2008) Light regulation and daytime dependency of inducible plant defenses in Arabidopsis: phytochrome signaling controls systemic acquired resistance rather than local defense. Plant Physiol. 147, 790-801

57. Zhang, L. et al. (2019) Comparison of transcriptome differences in soybean response to soybean mosaic virus under normal light and in the shade. Viruses 11, 793
58. Mirzahosseini, Z. et al. (2020) Comparative physiological and proteomic analysis of Arabidopsis thaliana revealed differentia wound stress responses following the exposure to different LED light sources. Environ. Exp. Bot. 169, 103895

59. Haga, K. and lino, M. (2004) Phytochrome-mediated transcriptional up-regulation of ALLENE OXIDE SYNTHASE in rice seedlings. Plant Cell Physiol. 45, 119-128

60. El-Esawi, M. et al. (2017) Blue-light induced biosynthesis of ROS contributes to the signaling mechanism of Arabidopsis cryptochrome. Sci. Rep. 7, 13875

61. Halder, V. et al. (2019) Plant chemical genetics reveals colistin sulphate as a SA and NPR1-independent PR1 inducer functioning via a p38-like kinase pathway. Sci. Rep. 9, 11196

62. Makowski, W. et al. (2019) Is a blue-red light a good elicitor of phenolic compounds in the family Droseraceae? A comparative study. J. Photochem. Photobiol. B Biol. 201, 111679

63. Caarls, L. et al. (2015) How salicylic acid takes transcriptional control over jasmonic acid signaling. Front. Plant Sci. 6, 170

64. Hayashi, M. and Kinoshita, T. (2011) Crosstalk between bluelight- and ABA-signaling pathways in stomatal guard cells. Plant Signal. Behav. 6, 1662-1664

65. Zhao, X. et al. (2007) A study of gibberellin homeostasis and cryptochrome-mediated blue light inhibition of hypocotyl elongation. Plant Physiol. 145, 106-118

66. Dillon, F.M. et al. (2018) Field-grown soybean induces jasmonates and defensive compounds in response to thrips feeding and solar UV-B radiation. Environ. Exp. Bot. 156, 1-7

67. Mannucci, A. et al. (2020) Hormone profile changes occur in roots and leaves of Micro-Tom tomato plants when exposing the aerial part to low doses of UV-B radiation. Plant Physiol. Biochem. 148, 291-301

68. Escobar Bravo, R. et al. (2019) Ultraviolet radiation enhances salicylic acid-mediated defense signaling and resistance to Pseudomonas syringae DC3000 in a jasmonic acid-deficient tomato mutant. Plant Signal. Behav. 14, e1581560

69. Prieto-Ruiz, I. et al. (2019) Supplementary UV radiation on eggplants indirectly deters Bemisia tabaci settlement without altering the predatory orientation of their biological contro agents Nesidiocoris tenuis and Sphaerophoria rueppellii. J. Pest. Sci. 92, 1057-1070

70. Vandenbussche, F. et al. (2018) An ultraviolet B condition that affects growth and defense in Arabidopsis. Plant Sci. 268 $54-63$

71. Cortés, L.E. et al. (2016) Trading direct for indirect defense? Phytochrome B inactivation in tomato attenuates direct antiherbivore defenses whilst enhancing volatile-mediated attraction of predators. New Phytol. 212, 1057-1071

72. Kegge, W. et al. (2013) Canopy light cues affect emission of constitutive and methyl jasmonate-induced volatile organic compounds in Arabidopsis thaliana. New Phytol. 200, 861-874

73. Kegge, W. et al. (2015) Red:far-red light conditions affect the emission of volatile organic compounds from barley (Hordeum vulgare), leading to altered biomass allocation in neighbouring plants. Ann. Bot. 115, 961-970

74. Campos, M.L. et al. (2016) Rewiring of jasmonate and phytochrome B signalling uncouples plant growth-defense tradeoffs. Nat. Commun. 7, 12570

75. Liu, C.C. et al. (2018) The bZip transcription factor HY5 mediates CRY1a-induced anthocyanin biosynthesis in tomato. Plant Cell Environ. 41, 1762-1775

76. Taulavuori, K. et al. (2016) Species-specific differences in synthesis of flavonoids and phenolic acids under increasing periods of enhanced blue light. Environ. Exp. Bot. 121, 145-150

77. Acharya, J. et al. (2016) Effects of light-emitting diode treatments on Brevicoryne brassicae performance mediated by secondary metabolites in Brussels sprouts. J. Plant Dis. Prot 123, 321-330

78. Zheng, C et al. (2019) Regulation of growth and flavonoid formation of tea plants (Camellia sinensis) by blue and green light. J. Agric. Food Chem. 67, 2408-2419

79. Fu, X. et al. (2015) Regulation of formation of volatile compounds of tea (Camellia sinensis) leaves by single light wavelength. Sci. Rep. 5, 16858 
80. Chakraborty, M. et al. (2019) Functional interrelation of MYC2 and HY5 plays an important role in Arabidopsis seedling development. Plant J. 99, 1080-1097

81. Dicke, M. and Lucas-Barbosa, D. (2020) Herbivore-induced plant volatiles as a source of information in plant-insect networks. In Biology of Plant Volatiles (2nd edn) (Pichersky, E. and Dudareva, N., eds), pp. 327-346, CRC Press

82. Zhang, Y. et al. (2020) Combined transcriptome and metabolome analysis identifies defence responses in spider miteinfested pepper (Capsicum annuum). J. Exp. Bot. 71, 330-343

83. Stukenberg, N. and Poehling, H.M. (2019) Blue-green opponency and trichromatic vision in the greenhouse whitefly (Trialeurodes vaporariorum) explored using light emitting diodes. Ann. Appl. Biol. 175, 146-163

84. Kim, K.N. et al. (2019) Advances in insect phototaxis and application to pest management: a review. Pest Manag. Sci. 75 , 3135-3143

85. Liu, Q. et al. (2019) Visual response effects of western flower thrips manipulated by different light spectra. Int. J. Agric. Biol. Eng. 12, 21-27

86. Park, J.H. and Lee, H.S. (2017) Phototactic behavioral response of agricultural insects and stored-product insects to light-emitting diodes (LEDs). Appl. Biol. Chem. 60, 137-144

87. Döring, T.F. and Röhrig, K. (2016) Behavioural response of winged aphids to visual contrasts in the field. Ann. Appl. Biol. $168,421-434$

88. Murata, M. et al. (2018) In the presence of red light, cucumber and possibly other host plants lose their attractability to the melon thrips Thrips palmi (Thysanoptera: Thripidae). Appl. Entomol. Zool. 53, 117-128

89. Suzuki, T. et al. (2008) Effects of light quality and intensity on diapause induction in the two-spotted spider mite, Tetranychus urticae. Appl. Entomol. Zool. 43, 213-218

90. Kaur, J. and Kariyat, R. (2020) Role of trichomes in plant stress biology. In Evolutionary Ecology of Plant-Herbivore Interaction (Núñez-Farfán, J. and Valverde, P.L., eds), pp. 15-35, Springe

91. Shibuya, T. et al. (2010) Preference of sweetpotato whitefly adults to cucumber seedlings grown under two different light sources. Hort. Technol. 20, 873-876

92. Ballaré, C.L. (2014) Light regulation of plant defense. Annu. Rev. Plant Biol. 65, 335-363

93. Rechner, O. et al. (2017) Can narrow-bandwidth light from UV-A to green alter secondary plant metabolism and increase Brassica plant defenses against aphids? PLoS One 12, e0188522

94. Qi, J. et al. (2018) Ultraviolet-B enhances the resistance of multiple plant species to lepidopteran insect herbivory through the jasmonic acid pathway. Sci. Rep. 8, 277

95. Velthuis, H.W. and Van Doorn, A. (2006) A century of advances in bumblebee domestication and the economic and environ mental aspects of its commercialization for pollination. Apidologie 37, 421-451

96. van Lenteren, J.C. et al. (2018) Biological control using invertebrates and microorganisms: plenty of new opportunities. BioControl 63, 39-59

97. Peitsch, D. et al. (1992) The spectral input systems of hymenopteran insects and their receptor-based colour vision. J. Comp. Physiol. A. 170, 23-40

98. Cochard, P. et al. (2019) The proportion of blue light affects parasitoid wasp behavior in LED-extended photoperiod in greenhouses: increased parasitism and offspring sex ratio bias. Biol. Control 133, 9-17

99. Briscoe, A.D.L.C. and Chittka, L. (2001) The evolution of color vision in insects. Annu. Rev. Entomol 46, 471-510
100. de Vries, L.J. et al. (2020) Bumblebees land remarkably well in red-blue greenhouse LED light conditions. Biol. Open 9, bio046730

101. Chu, C.-C. et al. (2009) Plastic cup traps equipped with lightemitting diodes for monitoring adult Bemisia tabaci (Homoptera: Aleyrodidae). J. Econ. Entomol. 96, 543-546

102. Johansen, N.S. et al. (2011) In the light of new greenhouse technologies. 2. Direct effects of artificial lighting on arthropods and integrated pest management in greenhouse crops. Ann Appl. Biol. 159, 1-27

103. SharathKumar, M. et al. (2020) Vertical farming: moving from genetic to environmental modification. Trends Plant Sci. 25 $724-727$

104. Zhang, M. and Runkle, E.S. (2019) Regulating flowering and extension growth of poinsettia using red and far-red lightemitting diodes for end-of-day lighting. HortScience 54, 323-327

105. Zhen, S. and Bugbee, B. (2020) Far-red photons have equivalent efficiency to traditional photosynthetic photons: implications for redefining photosynthetically active radiation. Plant Cell Environ. 43, 1259-1272

106. Van Lenteren, J.C. (2000) A greenhouse without pesticides: fact or fantasy? Crop Prot. 19, 375-384

107. Morandin, L.A. et al. (2001) Effect of bumble bee (Hymenoptera: Apidae) pollination intensity on the quality of greenhouse tomatoes. J. Econ. Entomol. 94, 172-179

108. Kaiser, E. et al. (2019) Adding blue to red supplemental light increases biomass and yield of greenhouse-grown tomatoes, but only to an optimum. Front. Plant Sci. 9, 2002

109. Bugbee, B. (2016) Toward an optimal spectral quality for plant growth and development: the importance of radiation capture. Acta Hortic. 1134, 1-12

110. Yadav, V. et al. (2005) A basic helix-loop-helix transcription factor in Arabidopsis, MYC2, acts as a repressor of blue light-mediated photomorphogenic growth. Plant Cell 17. 1953-1966

111. van Gelderen, K. et al. (2018) Far-red light detection in the shoot regulates lateral root development through the HY5 transcription factor. Plant Cell 30, 101-116

112. Pedmale, U.V. et al. (2016) Cryptochromes interact directly with PIFs to control plant growth in limiting blue light. Cell 164 , 233-245

113. Ortigosa, A. et al. (2020) The JA-pathway MYC transcription factors regulate photomorphogenic responses by targeting HY5 gene expression. Plant J. 102, 138-152

114. Casal, J.J. (2013) Photoreceptor signaling networks in plant responses to shade. Annu. Rev. Plant Biol. 64, 403-427

115. Kanazawa, A. and Kramer, D.M. (2017) In vivo modulation of nonphotochemical exciton quenching (NPQ) by regulation of the chloroplast ATP synthase. Proc. Natl. Acad. Sci. U. S. A. 99, 12789-12794

116. Takahashi, S. and Badger, M.R. (2011) Photoprotection in plants: a new light on photosystem II damage. Trends Plant Sci. 16, 53-60

117. Külheim, C. and Jansson, S. (2005) What leads to reduced fitness in non-photochemical quenching mutants? Physiol. Plant. 125, 202-211

118. Kromdijk, J. et al. (2016) Improving photosynthesis and crop productivity by accelerating recovery from photoprotection. Science 354, 857-861

119. Acevedo, F.E. et al. (2015) Cues from chewing insects - the intersection of DAMPs, HAMPs, MAMPs and effectors. Curr. Opin. Plant Biol. 26, 80-86 